\title{
Neurosarcoidosis of the conus medullaris and cauda equina presenting as paraparesis: case report and literature review
}

\author{
A Ku ${ }^{1}$, E Lachmann ${ }^{1}$, R Tunkel $^{2}$ and W Nagler ${ }^{1}$ \\ ${ }^{1}$ New York Hospital - Cornell Medical Center; and ${ }^{2}$ Memorial Sloan-Kettering Cancer Center, New York, USA
}

Sarcoidosis is a multi-system, non-caseating granulomatous disease of unknown etiology. Although spinal cord involvement is rare, it may cause severe neurological complications. We report the case of a 52-year-old woman who developed low back pain and a progressive flaccid paraparesis whose investigations revealed pulmonary and spinal sarcoidosis. To our knowledge, this is the first reported case of sarcoidosis of the conus medullaris and cauda equina diagnosed by an unusual enhancement pattern observed on magnetic resonance imaging with gadolinium. Biopsy of the lumbar nerve roots and of the lung revealed a noncaseating granuloma. Treatment with steroids and intensive rehabilitation resulted in remarkable functional improvement. Although rare, sarcoidosis of the conus medullaris and cauda equina should be considered in the differential diagnosis of flaccid paraparesis, particularly in patients with non-diagnostic evaluations.

Keywords: neurosarcoidosis; conus medullaris; cauda equina; paraparesis

\section{Introduction}

Sarcoidosis commonly affects the lung, skin, liver, lymph nodes and spleen. ${ }^{1}$ Five percent of all patients with sarcoidosis may develop neurosarcoidosis, affecting predominantly the basilar meninges or the cranial nerves (especially the facial nerve). ${ }^{2,3}$ Spinal cord sarcoidosis is rare and is usually preceded by a history of systemic sarcoidosis. ${ }^{4}$ Occasionally, spinal sarcoidosis can be the initial manifestation of the disease, without known systemic involvement. ${ }^{3,5-27}$ Upon review of these cases of neurosarcoidosis affecting the spinal cord, we find that less than $10 \%$ have involvement of the cauda equina. Our patient is unique for several reasons. First, flaccid paraparesis secondary to sarcoidosis of both the conus medullaris and cauda equina without known disseminated disease is rare. Second, magnetic resonance imaging with gadolinium diethylene triamine pentaacetic acid (MRI Gd-DTPA) was instrumental in making the correct diagnosis in our patient. Finally, prior reports on cauda equina sarcoidosis lack emphasis on the role of rehabilitation in the management of the paraparesis. ${ }^{5-7}$

\section{Case report}

A 52-year-old African-American woman was admitted for evaluation of a 6 months history of low back pain and progressive paraparesis. Her symptoms began with low back and right buttock pain. This progressed to

Correspondence: A Ku numbness and weakness of both legs. Investigative studies carried out in another hospital one month after the onset of her symptoms were unrevealing, including a normal myelogram. Two weeks prior to this admission, she was unable to walk due to progressive lower extremity weakness. She denied a history of bowel and bladder symptoms. Her past medical history was significant for a history of tuberculosis 30 years ago for which she was treated with Isoniazid. The family history was remarkable because of a brother who has Waldenstrom's macroglobulinemia.

On admission, the general physical examination was normal. Mental status examination and cranial nerve testing were unremarkable. Anal wink was absent but there was no saddle anesthesia present on testing. The patient was unable to stand because of symmetrical lower extremity weakness, greater proximally than distally. The iliopsoas, glutei and hamstrings were $4 / 5$, the quadriceps were $3 / 5$, and the tibialis anterior, extensor hallucis longus, gastrocnemius and soleus muscles were $2 / 5$ in strength. The knee and ankle reflexes were absent.The plantar responses were flexor. There was decreased sensation to pinprink in a stocking distribution in the lower extremities to the mid-thigh level. Vibration and position sense in both feet were intact.

Computed tomography (CT) of head and thoracolumbosacral spine as well as a bone scan were normal. A chest X-ray was significant showing a right apical density consistent with old pleural thickening without hilar adenopathy. Nerve conduction studies in the lower extremities were normal. Electromyography 
revealed evidence of denervation in the bilateral tibialis anterior, gastrocnemius and L4/5 and L5/S1 paraspinal musculature.

Significant findings on cerebrospinal fluid studies included an elevated protein content of $229 \mathrm{mg} / \mathrm{dL}$ (normal $<45 \mathrm{mg} / \mathrm{dL}$ ), leukocytosis (45 white blood cells), normal glucose level and negative cultures for bacteria, fungi, acid-fast bacilli and Lyme disease. Other cytopathological studies were negative. $B_{2}$ microglobulin levels were elevated. Oligoclonal bands were present.

Serum angiotensin converting enzyme level was at the upper range of normal at 21.2 (normal range: 6.1-21.2 units/L). Serum VDRL, ANA, ds DNA, thyroid function tests, cryptococcal antigen, HIV tests and Lyme titers were all within normal limits. The erythrocyte sedimentation rate was normal at $10 \mathrm{~mm}$ / $\mathrm{H}$.

MRI of the L-S spine revealed narrowing of the L5/ $\mathrm{S} 1$ interspace without disc herniation. However, MRI Gd-DPTA revealed subtle enhancement along the ventral and dorsal aspects of conus medullaris and the nerve roots of the cauda equina symmetrically, suggesting subarachnoid pathology. (Figure 1) No large nodular lesions were detected.

Neurosurgeons were consulted for a biopsy of the leptomeninges, spinal nerves and dura mater in the region of leptomeningeal enhancement seen on MRI. Pathology revealed fibrous thickening of the meninges, degenerating nerve roots with inflammatory cells, reactive lymphocytes and non-caseating granulomata (Figure 2). Bronchial mycology cultures were negative. Cerebrospinal, pulmonary and urine AFB cultures were negative. The gallium scan revealed an increased uptake in the bilateral perihilar lymph node region. Bronchoscopy with biopsy confirmed the diagnosis of sarcoidosis, showing multinucleated giant cells without caseation. (Figures 3 and 4).

Daily oral Prednisone $50 \mathrm{mg}$ and physical therapy were initiated for the management of her neurosarcoidosis. Functional evaluation revealed severe deficits in static standing balance secondary to poor ankle control resulting from bilateral foot-drop. The patient also had difficulty in the dressing of her lower limbs. Bilateral AFOs and assistive devices were utilized for transfer, gait, static and dynamic standing balance and stair training. The patient improved with daily therapy and by the time of discharge 1 month later, she was able to ambulate 500 feet independently with bilateral AFOs and Lofstrand crutches. She was also independent for her activities of daily living.

Over the next 6 months the patient improved functionally whilst taking low dose oral Prednisone. She was able to ambulate with a pair of Lofstrand crutches independently for 700 feet and returned to part-time employment. Four years later, both the sarcoidosis and her neurological deficits stabilized on maintenance low dose prednisone and weekly physical and hydrotherapy. Her old pulmonary tuberculosis did not reactivate during the four years on steroid. The
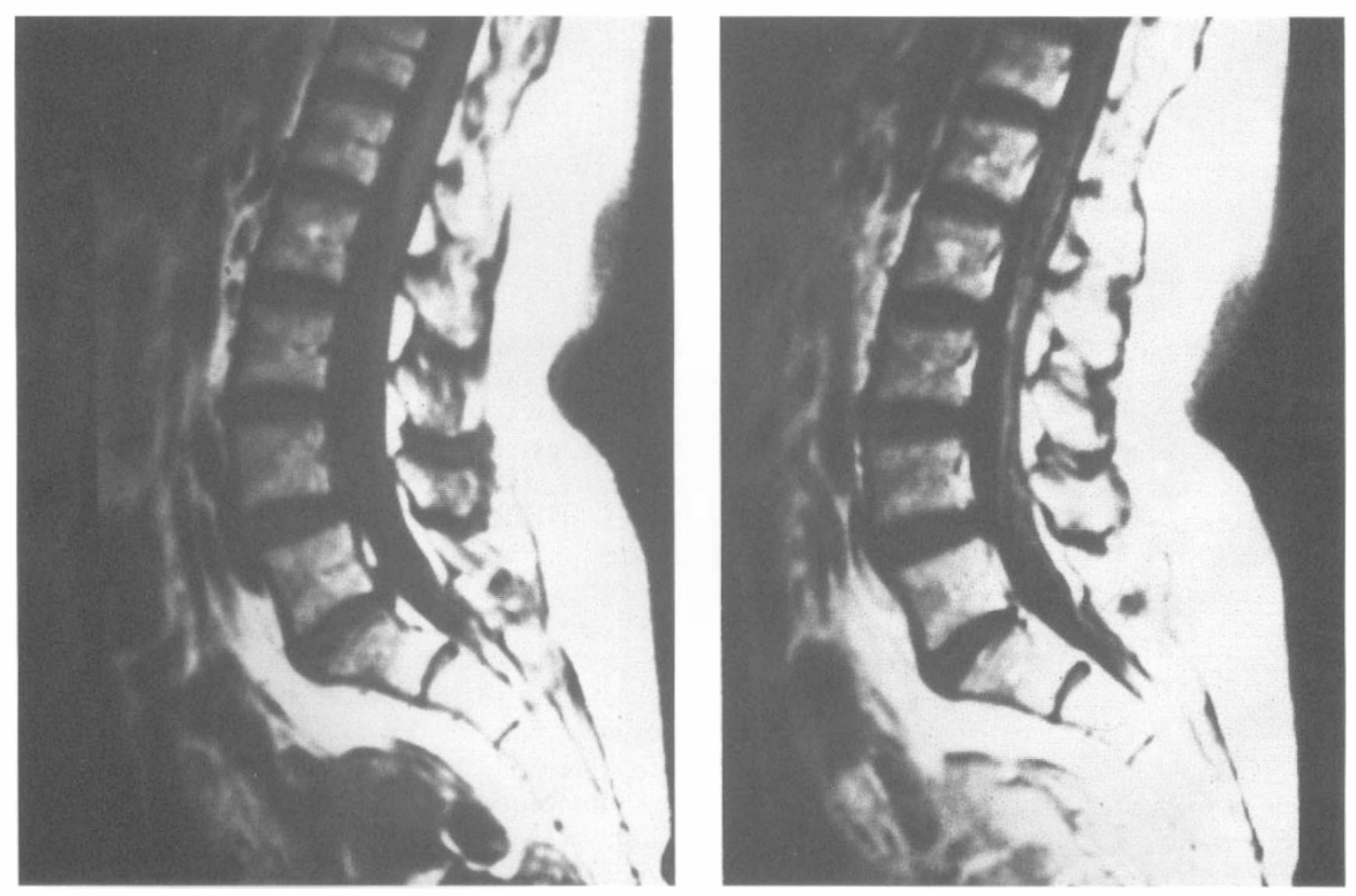

Figure 1 Left: MRI without contrast. Right: MRI with Gadolinium demonstrating enhancement along the ventral and dorsal aspects of the conus medullaris and the nerve root of the cauda equina 


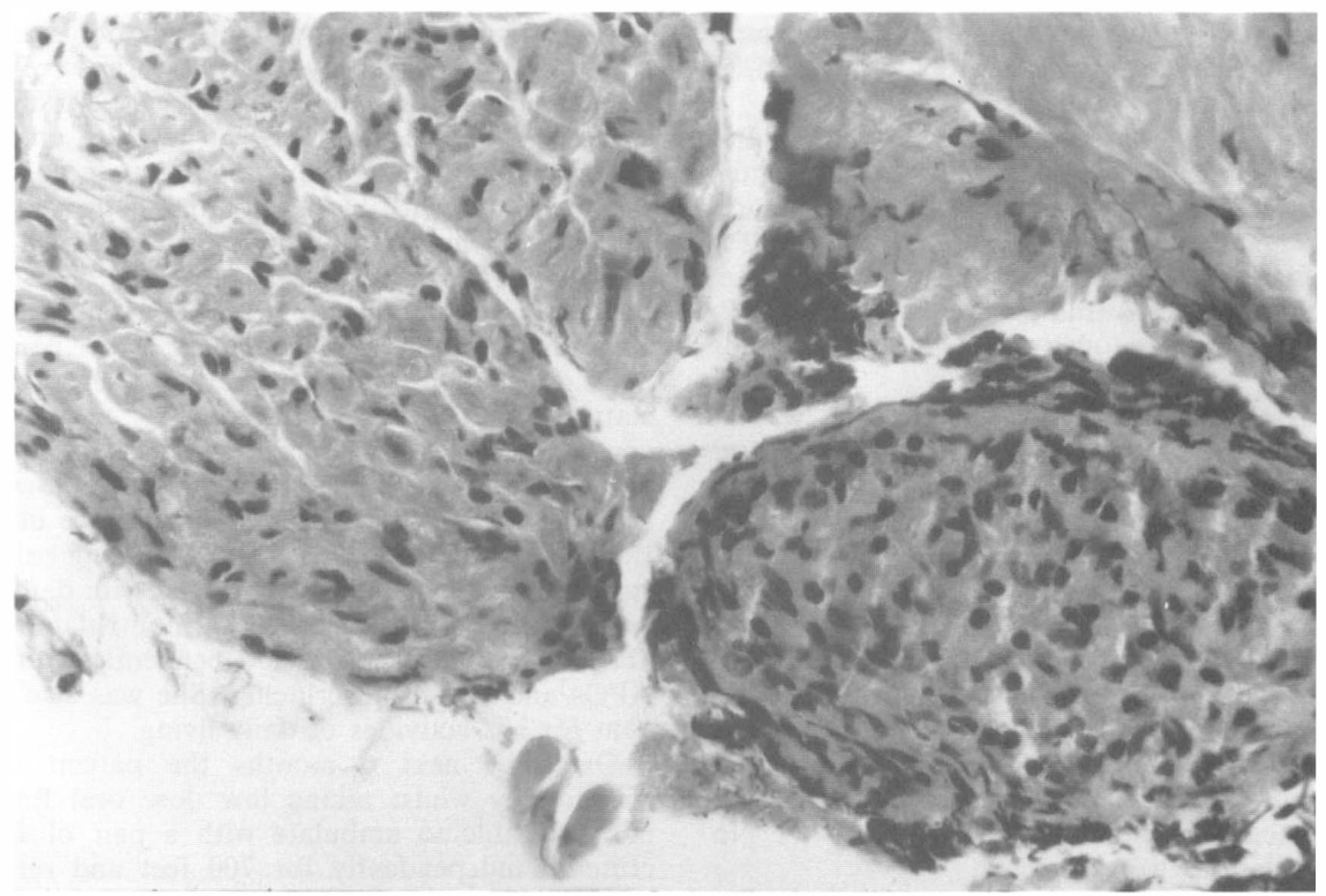

Figure 2 Cauda equina biopsy demonstrating a non-caseating granuloma on the right lower quadrant

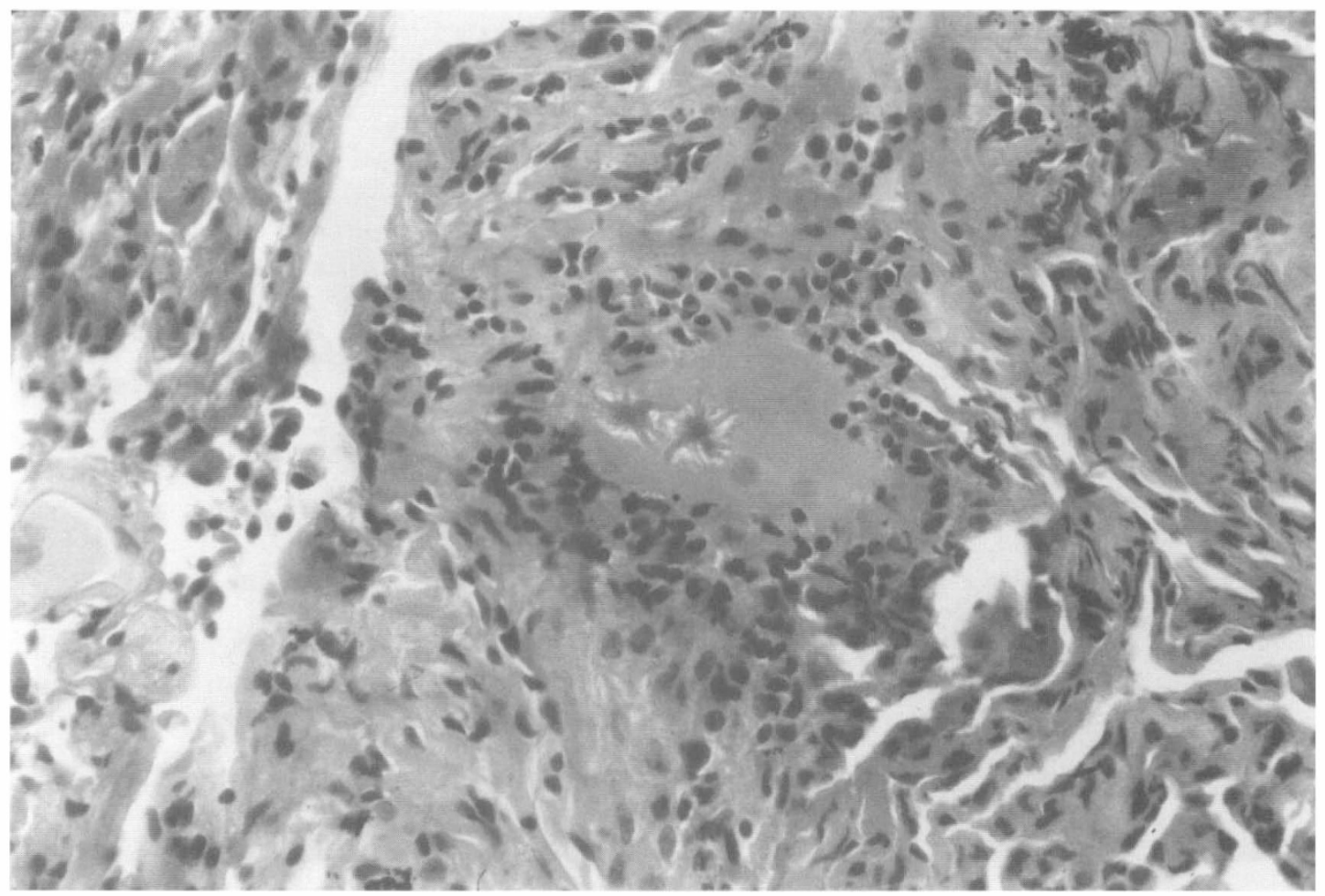

Figure 3 Pulmonary biopsy demonstrating a non-caseating granuloma with an asteroid body in the center 


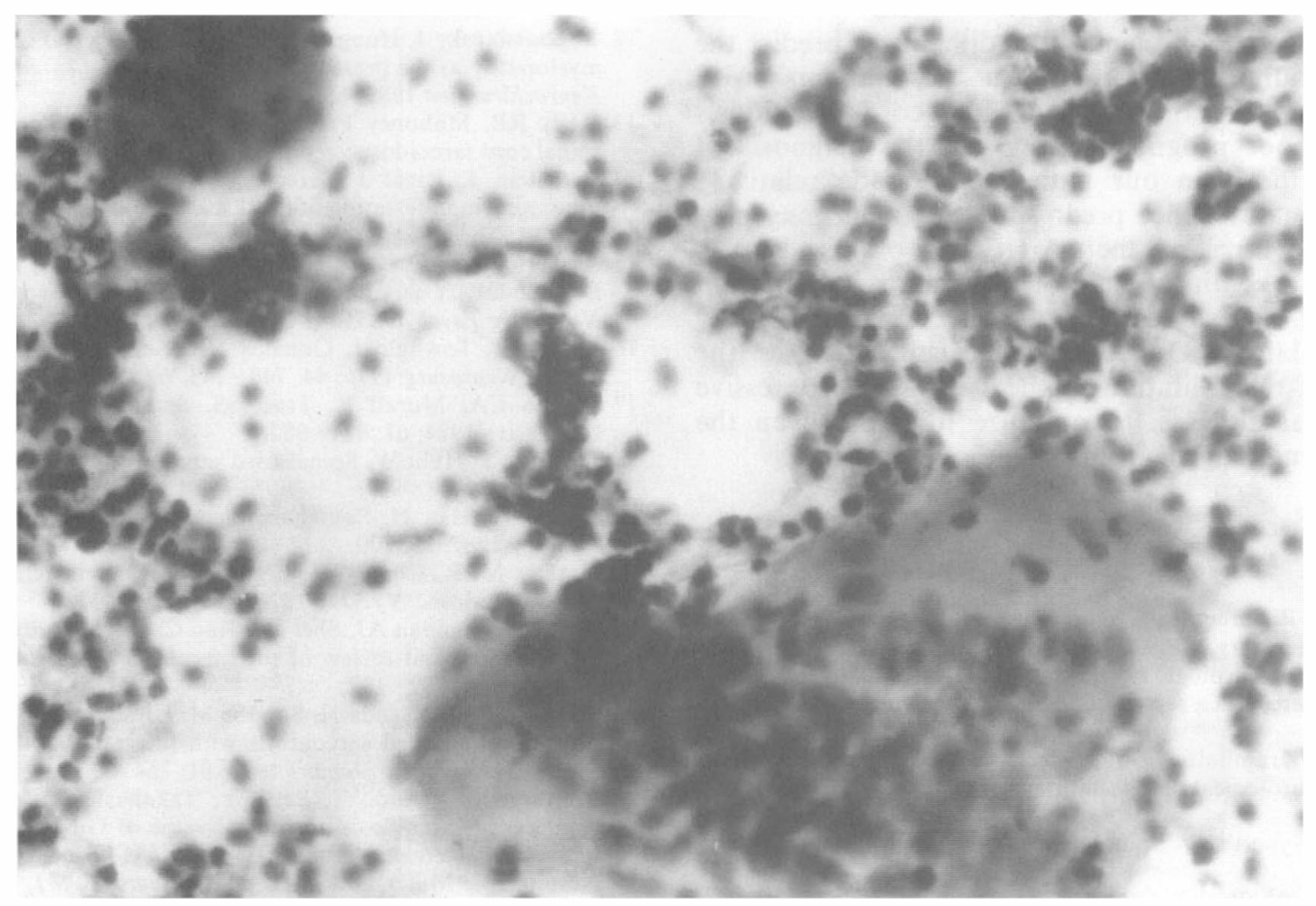

Figure 4 Pulmonary biopsy showing a giant cell

bilateral lower limb weakness has persisted as compared to our initial evaluation four years ago. However, the patient enjoys a good quality of life despite the physical deficits. She drives a car, and is able to function in the community with the use of a quad-cane and bilateral AFOs while walking.

\section{Discussion}

Of the patients whose initial manifestation of sarcoidosis was a cauda equina syndrome, all had abnormalities on myelography which eventually led to the diagnosis of sarcoidosis. ${ }^{5-7}$ Our patient's myelogram did not reveal any filling defects, reflecting the low sensitivity of myelogram in detecting smaller sarcoid lesions. In a patient with a history of sarcoidosis who later developed a cauda equina syndrome, signal intensity changes may be present on non-contrast MRI. ${ }^{6}$ However, the conus lesion in that patient was presumed to be sarcoid, but was not biopsy-proven. In our patient, the abnormalities of the cauda equina and conus medullaris, not detected on myelography or non-contrast MRI, were eventually visualized on MRI Gd-DTPA. The inflammation of the early stage of spinal cord sarcoidosis may correspond to this pattern of leptomeningeal enhancement on MRI Gd-DTPA. ${ }^{25}$

MRI, showing signal intensity changes with sarcoid tissue, is being used increasingly to assist in the diagnosis and management of spinal cord sarcoidosis. ${ }^{10.13 .22-28}$ The presence of patchy, multifocal parenchymal enhancement and areas of linear peripheral enhancement was observed on MRI Gd-DTPA. ${ }^{24}$ In one series, enhancement was determined to be a helpful clue in the diagnosis of neurosarcoidosis in 15 of 17 patients. ${ }^{28}$ Meanwhile, MRI has also been used to monitor progress with treatment. ${ }^{26,28}$ Regression of central nervous abnormalities after steroid treatment were noted on follow-up MRI Gd-DTPA. ${ }^{27,28}$

Despite the high sensitivity of MRI, the enhancement patterns seen with sarcoidosis on MRI GdDTPA is not specific for sarcoidosis. Myelitis, multiple sclerosis, and malignancy may produce similar enhancement on MRI. ${ }^{10,13,24}$ Other conditions to exclude are HIV myelopathy and fungal or tuberculous granulomata. Prior to the spinal biopsy in our patient, there was no clinical evidence for sarcoidosis involvement outside the central nervous system. Thus, the optimal diagnosis of spinal cord sarcoidosis requires histological confirmation. Unfortunately, morbidity and mortality can be associated with a spinal cord biopsy. ${ }^{25}$ For this reason, in the proper clinical setting, abnormal findings on MRI Gd-DTPA in patients with established systemic sarcoidosis may make spinal biopsy unnecessary.

Corticosteroids are the treatment of choice in most patients with neurosarcoidosis. However, there is no established dosage or duration of corticosteroid 
treatment in these patients. It is difficult to predict the clinical course with treatment of patients with neurosarcoidosis. In general, spinal cord sarcoidosis carries a poor prognosis. However, the diagnosis of neurosarcoidosis in our patient was made relatively early and maintenance prednisone treatment may play a role in controlling the progression of her disease. Furthermore, with the help of on-going rehabilitation, the patient has successfully avoided loss of mobility and ambulation. Our patient clearly illustrates the purpose of rehabilitation in the setting of progressive neurological diseases: to optimize function given the level of remaining neuromuscular capacity.

\section{References}

1 Sharma OP. Sarcoidosis: clinical, laboratory and immunologic aspects. Seminars in Roentgenology 1985; 20: $340-355$.

2 Delaney P. Neurologic manifestations in sarcoidosis: review of the literature with a report of 23 cases. Ann Intern Med 1977; 87: $336-345$.

3 Stern BJ, Krumholz A, Johns C, Scott P, Nissim J. Sarcoidosis and its neurological manifestations. Arch Neurol 1985; 42: $909-$ 917.

4 Day AL, Sypert GW. Spinal cord sarcoidosis. Ann Neurol 1977; 1: $79-85$.

5 Campbell JN, Black P, Ostrow PT. Sarcoid of the cauda equina. $J$ Neurosurg 1977; 47: 109-112.

6 Zajicek J. Sarcoidosis of the cauda equina: a report of three cases. J Neurol 1990; 237: 424-426.

7 Emery JP, Lasserre PP, Dryll A. Les manifestations neurologiques peripheriques de la sarcoidosis. Sem Hop Paris 1972; 48: $3039-3043$.

8 Graf M, Wakhloo A, Schmidtke K, Bloss H, Volk B. Sarcoidosis of the spinal cord and medulla oblongata. A pathological and neuroradiological case report. 1994; 13: $19-25$

9 Tuel SM, Meythaler JM, Cross LL. Rehabilitation of quadriparesis secondary to spinal cord sarcoidosis. Am J Phys Med Rehabil 1991; 70: 63-69.

10 Kayama S, Kikuchi S. Intramedullary spinal cord sarcoidosis. Spine 1993; 18: $2118-2120$.

11 Cahill DW, Salcman M. Neurosarcoidosis: a review of the rarer manifestations. Surg Neurol 1981; 15: 204-211.
12 Bogousslavsky J, Hungerbuhler JP, Regli F, Graf HJ. Subacute myelopathy as the presenting manifestation of sarcoidosis. Acta Neurochirurgica 1982; 65: $193-197$.

13 Kelly RB, Mahoney PD, Cawley KM. MR demonstration of spinal cord sarcoidosis: report of a case. AJNR 1988; 9: 197-199.

14 Bernstein J, Rival J. Sarcoidosis of the spinal cord as the presenting manifestation of the disease. Southern Med J 1978: 71 : $1571-1573$.

15 Vighetto A, Fischer G, Collet P, Bady B, Trillet M. Intramedullary sarcoidosis of the cervical spinal cord. $J$ Neurol Neurosurg Psych 1985; 48: 477-479.

16 Snyder R, Towfighi J, Gonatas NK. Sarcoidosis of the spinal cord. J Neurosurg 1976; 44: $740-743$.

17 Martin CA, Murali R, Trasi SS. Spinal cord sarcoidosis. J Neurosurg 1984; 61: $981-982$.

18 Banerjee T, Hunt W. Spinal cord sarcoidosis. J Neurosurg 1972; 36: $490-493$.

19 Huang H, Haq N. Spinal leptimeningeal sarcoidosis. Neuroradiology 1987; 29: 100.

20 Nathan MPR, Chase PH, Elguezabel A, Weinstein M. Spinal cord sarcoidosis. NYSJM 1976; May: $748-752$.

21 Fried ED, Landau AJ, Sher JH, Rao C. Spinal cord sarcoidosis: a case report and review of the literature. J Assoc Acad Minor Phys 1993; 4: $132-137$.

22 Yamashita M, Yasuda H, Sonobe M, Hisanaga T, Shigeta Y. A case of spinal cord sarcoidosis with interesting MRI findings. Rinsho Shinkeigaku (Japan) 1991; 31: 734-737.

23 Kameyama T, Ando T, Saito Y, Takahashi A, Ymamda H. Spinal cord sarcoidosis-diagnostic value of Gd-enhanced MRI Rhinsho Shinkeigaku (Japan) 1992; 32: 631-636.

24 Nesbit GM, Miller GM, Baker HL, Ebersold MJ, Scheithauer BW. Spinal cord sarcoidosis: a new finding at MR imaging with Gd-DTPA enhancement. Radiology 1989; 173: 839-843.

25 Junger SS, Stern BJ, Levine SR, Sipos E, Marti-Masso JF. Intramedullary spinal sarcoidosis: clinical and magnetic resonance imaging characteristics. Neurology 1993; 43: 333-337.

26 Sauter MK, Panitch HS, Kristt DA. Myelopathic neurosarcoidosis: diagnostic value of enhanced MRI. Neurology 1991; 41: $150-151$.

27 Morita H, Hasyashi R, Tako K, Tsukada N, Yanagisawa N. Spinal cord sarcoidosis: MRI findings in response to treatment. Eur Neurol 1992; 32: 126-129.

28 Lexa FJ, Grossman RI. MR of sarcoidosis in the head and spine: spectrum of manifestations and radiographic response to steroid therapy. Am J Neuroradiology 1994; 15: $973-982$. 